\title{
Zur Glykogenbildung in der Leber aus Formaldehyd.
}

Von

Karl Grube.

Die Versuche ${ }^{1}$ ), bei denen eine stark verdünnte Formaldehydlösung lange Zeit durch die überlebende Schildkrötenleber durchgeleitet worden war, hatten ergeben, dass die Leberzelle diese einfachsten Moleküle zur Glykogenbildung zu verwerten vermochte.

Wie dabei der Vorgang sich im einzelnen abspielt, entzieht sich vorläufig unserer Kenntnis; doch ist darauf aufmerksam zu machen, dass nach Untersuchungen von $H$. und A. Euler ${ }^{2}$ ) der Formaldehyd in starker Verdünnung bei sehwach alkalischer, ja praktisch neutraler Reaktion leicht zu Zucker kondensiert werden kann. Es liegt daher die Möglichkeit vor, dass der Vorgang in der Leber analog verläuft, da hier die Verhältnisse, welche die beiden Autoren als für die Kondensierung des Formaldehyds zu Zucker als günstig nachgewiesen haben, vorliegen, nämlich : verdünnte Lösung von Formaldehyd und Anwesenheit von schwachen Alkalien in der Lösung. Es ist ferner von Interesse, dass die genannten Forscher als Zwischenprodukt den Glykolaldehyd nachweisen konnten. Aus dem so durch Kondensierung entstandenen Zucker könnte dann die Leberzelle das Glykogen bilden.

Man hat nun gegen meine Versuche den Einwand gemacht, dass das entstandene Glykogen nicht aus dem Formaldehyd stamme, sondern dass dieser nur als Reiz gewirkt habe, und dass die Leber

1) K. Grube, Über die kleinsten Moleküle, welche die Leber zur Synthese des Glykogenes verwerten kann. Pflüger's Arch. Bd. 121 S. 636. 1908.

2) H. und A. Euler, Zur Kenntnis der Zuckerbildung ans Formaldehyd. Chem. Ber. Bd. 39 S. 39. 1906. - Dieselben, Zur Kenntnis des Formaldehyds. Chem. Ber. Bd. 38 S. 2551. 1905. 
durch diesen Reiz zur Produktion von Zucker aus irgendeiner unbekannten Substanz veranlasst würde.

Wenn auch dieser Vorgang nicht gerade wahrscheinlich erscheint, so ist doch seine Möglichkeit nicht abzustreiten, und ich habe daher auf Vorschlag und Veranlassung von Herrn Geheimrat Pflüger eine Anzahl von Reizmitteln versucht und dieselben in derselben Weise anf die Leber einwirken lassen wie den Formaldehyd.

Es gelangten folgende Stoffe zur Verwendung: Phenol, Sublimat, salpetersaures Silber, Essigsäure, Kaliumbydrat, Ammoniumkarbonat.

Versuch I. Benutzt zur Durchleitung 10 Liter physiologiseher Kochsalzlösung, enthaltend $5 \mathrm{~g}$ Phenol $=0,05 \%$.

Rechter Leberlappen, Gewicht 12,56 g, enthält vor der Durchleitung $9,5 \%$ Glykogen.

Linker Leberlappen, Gewicht $34,38 \mathrm{~g}$, enthält nach der Durchleitung 5,5\% Glykogen.

$$
\text { Abnahme } 46,8^{\circ} \% \text {. }
$$

Versuch II. Benutzt zur Durchleitung 10 Liter physiologischer Kochsalzlösung, enthaltend $5 \mathrm{~g}$ Phenol $=0,05 \%$.

Rechter Leberlappen, Gewicht $19,14 \mathrm{~g}$, enthält vor der Durchleitung 8,54\% Glykogen.

Linker Leberlappen, Gewicht $26,43 \mathrm{~g}$, enthält nach der Durchleitung $4,7 \%$ Glykogen.

$$
\text { Abnahme 45,2\%. }
$$

Versuch III. Benutzt zur Durchleitung 10 Liter physiologischer Kochsalzlösung, enthaltend $2 \mathrm{~g}$ Sublimat $=0,02 \%$.

Rechter Leberlappen, Gewicht $13,4 \mathrm{~g}$, enthält vor der Durchleitung $9,54 \%$ Glykogen.

Linker Leberlappen, Gewicht $26,23 \mathrm{~g}$, enthält nach der Durchleitung $7,14 \%$ Glykogen.

$$
\text { Abnahme 25,2\%. }
$$

Versuch IV. Benutzt zur Durchleitung 10 Liter physiologischer Kochsalzlösung, enthaltend $2 \mathrm{~g}$ Sublimat $=0,02 \%$.

Rechter Leberlappen, Gewicht $9,45 \mathrm{~g}$, enthält vor der Durchleitung $0,21 \%$ Glykogen.

Linker Leberlappen, Gewicht $17,14 \mathrm{~g}$, enthält nach der Durchleitung $0,055 \%$ Glykogen. 
Versuch V. Benutzt zur Durchleitung 10 Liter 0,4\% iger Lösung von essigsaurem Natron, enthaltend $2 \mathrm{~g}$ salpetersaures Silber $=0,02 \%$.

Rechter Leberlappen, Gewicht $10,54 \mathrm{~g}$, enthält vor der Durchleitung $3,26 \%$ Glykogen.

Linker Leberlappen, Gewicht 14,51, enthält nach der Durchleitung $1,93 \%$ Glykogen.

$$
\text { Abnahme 40,8\%. }
$$

Versuch VI. Zur Durchleitung benutzt 10 Liter $0,4 \%$ iger Lösung von essigsaurem Natron, enthaltend $0,5 \mathrm{~g}$ salpetersaures Silber $=0,005 \%$.

Rechter Leberlappen, Gewicht $15,14 \mathrm{~g}$, enthält vor der Durchleitung 2,54\% Glykogen.

Linker Leberlappen, Gewicht $21,68 \mathrm{~g}$, enthält nach der Durchleitung $2,16 \%$ Glykogen.

$$
\text { Abnahme 14,9\%. }
$$

Versueh VII. Benutzt zur Durchleitung physiologisehe Kochsalzlösung, enthaltend $1 \mathrm{~g}$ Essigsäure $=0,01 \%$.

Rechter Leberlappen, Gewicht 18,69 g, enthält vor der Durchleitung 7,8\% Glykogen.

Linker Leberlappen, Gewicht $21,34 \mathrm{~g}$, enthält nach der Durchleitung $6,77 \%$ Glykogen.

$$
\text { Abnahme } 13,2 \% \text {. }
$$

Versuch VIII. Benutzt zur Durchleitung 10 Liter physiologischer Kochsalzlösung, enthaltend 0,5 g Essigsäure $=0,005 \%$.

Rechter Leberlappen, Gewicht $6,53 \mathrm{~g}$, enthält vor der Durchleitung 1,48\% Glykogen.

Linker Leberlappen, Gewicht 14,06 g, entbält nach der Durchleitung 1,12\% Glykogen:

$$
\text { Abnahme } 25 \% \text {. }
$$

Versuch IX. Benutzt zur Durchleitung 10 Liter Ringer'scher Lösung, enthaltend $2 \mathrm{~g}$ Kaliumbydrat $=0,02 \%$.

Rechter Leberlappen, Gewicht $8,34 \mathrm{~g}$, enthält vor der Durchleitung $0,32 \%$ Glykogen.

Linker Leberlappen, Gewicht 26,29 g, enthält nach der Durchleitung Spuren Glykogen.

Versuch X. Benutzt zur Durchleitung 10 Liter Ringer'scher Lösung, enthaltend $1 \mathrm{~g}$ Kaliumhydrat $=0,01 \%$. 
Rechter Leberlappen, Gewicht $9,06 \mathrm{~g}$, enthält vor der Durchleitung $3,89 \%$ Glykogen.

Linker Leberlappen, Gewicht 20,7 g, enthält nach der Durchleitung $3,15 \%$ Glykogen.

$$
\text { Abnahme } 19 \% \text {. }
$$

Versuch XI. Benutzt zur Durchleitung 10 Liter Ringer'scher Lösung, enthaltend $5 \mathrm{~g}$ Ammoniumkarbonat $=0,05 \%$.

Rechter Leberlappen, Gewicht $7,4 \mathrm{~g}$, enthält vor der Durchleitung 3,3\% Glykogen.

Linker Leberlappen, Gewicht $15,64 \mathrm{~g}$, enthält nach der Durehleitung 2,34\% Glykogen.

\section{Abnahme $29 \%$.}

Versuch XII. Benutzt zur Durchleitung 10 Liter Ringer'scher Lösung, enthaltend $1,2 \mathrm{~g}$ Ammoniumkarbonat $=0,02 \%$.

Rechter Leberlappen: Gewicht 13,04 g, enthält vor der Durchleitung $1,54 \%$ Glykogen.

Linker Leberlappen: Gewicht $15,6 \mathrm{~g}$, enthält nach der Durchleitung $1,06 \%$ Glykogen.

Abnahme 31,1\%.

Die Versuche beweisen zur Evidenz, dass Reizung der Leberzellen allein eine Glykogenbildung nicht hervorrufen kann.

Wie die meist beträchtliche Abnahme des Glykogengehaltes nach der Durchleitung zeigt, findet eine starke Inversion des Glykogens statt. Um festzustellen, wieviel davon durch die Durchleitung der grossen Flüssigkeitsmassen, wieviel durch die Reizstoffe hervorgerufen sei, wurde in einer Anzahl von Versuchen so verfahren, dass der eine (rechte) Leberlappen nur mit Ringer'scher Lösung durchströmt wurde, während durch den andern (linken) der betreffende Reizstoff floss.

Versuch XIII. Benutzt zur Durehleitung 6 Liter Ringerscher Lösung + 0,6 g Ammoniumkarbonat.

Rechter Leberlappen: Gewicht $11,24 \mathrm{~g}$, enthaltend nach der Durchleitung von Ring e r'scher Lösung ohne Zusatz 1,39\% Glykogen.

Linker Leberlappen: Gewicht $14,83 \mathrm{~g}$, enthält nach der Durchleitung der Ammoniumkarbonatlösung 1,04\% Glykogen. 
Versuch XIV. Benutzt zur Durchleitung 6 Liter Ringerscher Lösung + 0,6 g Ammoniumkarbonat.

Rechter Leberlappen: Gewicht $15 \mathrm{~g}$, enthält nach der Durchleitung von Ringer'scher Lösung ohne Zusatz 1,01\% Glykogen.

Linker Leberlappen: Gewicht 12,01 g, enthält nach der Durchleitung von $0,01 \%$ iger Ammoniumkarbonatlösung 0,927\% GlykogenAbnahme $8,2 \%$.

Versuch XV. Benutzt zur Durchleitung 6 Liter Ringerscher Lösung $+1,2 \mathrm{~g}$ Phenol $=0,02 \%$.

Rechter Leberlappen: Gewicht $10,3 \mathrm{~g}$, enthält nach der Durchleitung von Ringer'scher Lösung ohne Zusatz 0,22\% Glykogen.

Linker Leberlappen: Gewicht $10,4 \mathrm{~g}$, enthält nach der Durchleitung von $0,02 \%$ iger Phenollösung $0,129 \%$ GJykogen.

Abnahme $41,3 \%$.

Versuch XVI. Benutzt zur Durchleitung 6 Liter Ringerscher Lösung $+0,9 \mathrm{~g}$ Sublimat $=0,015 \%$.

Rechter Leberlappen: Gewicht $11,1 \mathrm{~g}$, entbält nach der Durchleitung von Ringer'scher Lösung ohne Zusatz Spuren Glykogen. Linker Leberlappen: Gewicht $7,29 \mathrm{~g}$, enthält nach der Durchleitung von 0,015.\% iger Sublimatlösung minimale Spuren Glykogen.

Die Inversion des Glykogens ist also grösser in dem Leberlappen, durch welchen der Reizstoff geleitet worden ist.

Herrn Geheimrat P flüger gestatte ich mir für die Ratschläge und die bei der Ausführung der Versuche gewährte Förderung meinen besten Dank auszusprechen. 